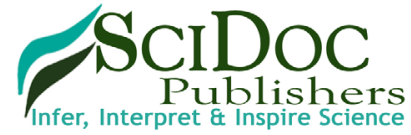

International Journal of Surgery and Research (IJSR)

ISSN 2379-156X

\title{
Upper Tract Urothelial Tumor with Renal Vein and Inferior Vena Cava Thrombus Case Report
}

Case Report

Raed $\mathrm{A}^{1^{*}}$, Alturki $\mathrm{A}^{2}$

${ }^{1}$ Sattam Bin Abdulaziz University, College of Medicine, Surgery Department, Saudi Arabia.

${ }^{2}$ King Saud University, College of Medicine, Saudi Arabia.

Abstract

Introduction: Urothelial tumors of upper tract are uncommon in clinical practice compared to other renal malignancy.

Presentation of Case: A 71-year-old man with multiple co-morbidities who presented with generalized non-specific symptoms was worked up for his abnormal lab results. On imaging he was found to have an enlarged left kidney with perinephric fat stranding and inferior vena cava thrombus. Biopsy of the thrombus showed morphological features characteristic of malignant tumors with a papillary growth pattern. Preoperative diagnosis pointed towards Renal Cellular Carcinoma with IVC thrombus. The patient underwent left radical nephrectomy and IVC thrombectomy. Surgical pathology of the retrieved specimens showed high-grade urothelial (transitional cell) carcinoma of the renal pelvis with multiple lymph nodes and high-grade urothelial carcinoma and high-grade papillary urothelial carcinoma of the IVC thrombus, tumor stage (AJCC $7^{\text {th }}$ edition): pT4b, N3, MX.

Discussion: vascular involvement make small portion of renal malignancy but with urothelial origin make it more rare and few cases are reported in literature.

Conclusion: To our knowledge this is the first reported case of upper tract urothelial cell carcinoma with IVC thrombus that mimics renal cell carcinoma in the Kingdom of Saudi Arabia and middle east.

\section{Introduction}

Renal malignancies are usually diagnosed incidentally, with imaging that is carried out for other reasons, long before the characteristic symptoms of the malignancy appear. Renal malignancies, mainly renal cell carcinoma (RCC), account for about $85 \%$ of kidney cancer, while the majority of the remaining $12 \%$ is composed of upper tract urothelial cancers [1]. The involvement of the renal vein/inferior vena cava has been reported in about $4-10 \%$ of RCC malignancies [2]. Now while the incidence of upper tract urothelial cancer with IVC thrombus has been reported a handful of times, to our knowledge this is the first reported case in the Kingdom of Saudi Arabia. This Case report has been reported in line with the SCARE criteria [10].

\section{Case Report}

A 71 year old man from Riyadh Saudi Arabia, known to have hypertension, diabetes mellitus type two, dyslipidemia, ischemic heart disease, congestive heart failure, and chronic kidney disease, presented to the ER with generalized symptoms of decreased oral intake, and nausea and vomiting all of which had started only 2 days prior. There was no history of hematuria, flank pain or lower urinary tract symptoms. Bedside examination of the patient showed mild abdominal distention and lower limb edema.

Lab findings showed hyponatremia and high creatinine, so the patient was originally admitted under internal medicine for management.

Abdominal and renal US were ordered to identify a cause for the patient's vomiting and abdominal distention. The studies showed an IVC thrombosis (FIGURE 1) which was present at the level of the hepatic vein, with the partial Superior Mesenteric Vein (SMV) thrombus (FIGURE 1). Both kidneys showed an increase in echogenicity, more in the left kidney with mild hydronephrosis and multiple cysts, but no solid masses could be appreciated.

An abdominal and pelvic CT, without contrast due to the patients comprised renal function, showed a left kidney, which was enlarged with surrounding fat stranding, but no definite discrete mass could be identified. The left renal vein was enlarged

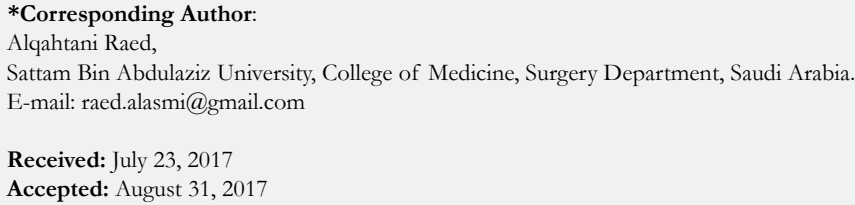

Copyright: Raed $\mathbf{A}^{\circ}$ 2017. This is an open-access article distributed under the terms of the Creative Commons Attribution License, which permits unrestricted use, distribution and reproduction in any medium, provided the original author and source are credited. 
secondary tumor thrombus extending to IVC (FIGURE 2). The infrahepatic IVC was involved by tumor thrombus extension as Level 3 thrombus (FIGURE 3). Distally, it was patent. It also showed multiple enlarged para-aortic and pre-aortic lymph nodes mainly in the left para-aortic region. The largest lymph node measured $1.9 \times 1.5 \mathrm{~cm}$.

Do to the limitation of non-enhanced CT and MRI and the lack of a discrete mass being identified in either study; the initial impression was a thrombus, which was not due to renal malignancy. Angiographic IVC biopsy was done to determine the origin of the thrombus. The biopsy result showed morphological features, which were characteristic of malignant tumors with a papillary growth pattern. After the result of the biopsy and due to the nature of the thrombus, a diagnosis of RCC was made. The patient was booked for angioembolization then left radical nephrectomy and IVC thrombectomy.

After successful induction of general anesthesia, the patient was kept in a supine position. Laparotomy incision was made and the peritoneum was opened, mobilization of the small bowl was done and the retroperitoneum was opened on the right side. The infra-hepatic part of the IVC was also exposed and skeletonized; the suprahepatic part of the IVC proved challenging to isolate, so a right subcostal incision had to be made and the liver was completely dissected and mobilized. Isolation of the infrarenal part of the IVC, right renal pedicle, and porta hepatis was done with vessel loops on all of them. Our attention was then directed towards the left kidney and we isolated its pedicle. All the four previous Penrose drains were tightened and all the vessels were controlled. The IVC and left renal veins were opened over the thrombus, and the thrombus was removed. The IVC was then closed and our attention was focused towards the left kidney which harbored the malignancy. It was then dissected completely and delivered outside the body. Finally lymph node dissection was done to the suspicious lymph nodes that were shown on the CT. Pathology report for the left kidney showed high-grade urothelial (transitional cell) carcinoma of the renal pelvis, invading through the kidney into perinephric fat, renal sinuses and renal hilar blood vessels. Sections from the specimen also showed multiple lymph nodes with high-grade urothelial carcinoma and necrosis. Tumor stage (AJCC $7^{\text {th }}$ edition): p'T4b, N3, MX.

\section{Discussion}

Incidence of renal malignancy is influenced by many factors such as race, gender, and geographical location. The incidence of renal malignancies has increased in the last two decades here in Saudi Arabia [3]. One reason for this could be due to the advancements in diagnostic imaging as well as its wider availability and its liberal

Figure 1. Abdominal US showing IVC and SMV thrombosis.

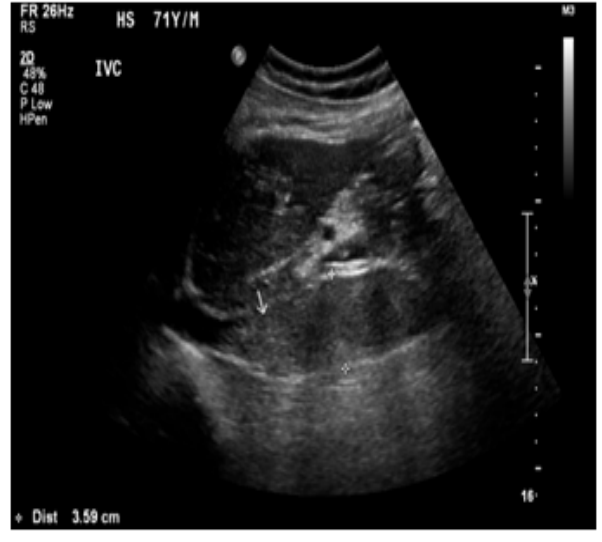

Figure 2. Non-enhanced CT showing an enlarged left kidney with fat stranding but no definitive mass.

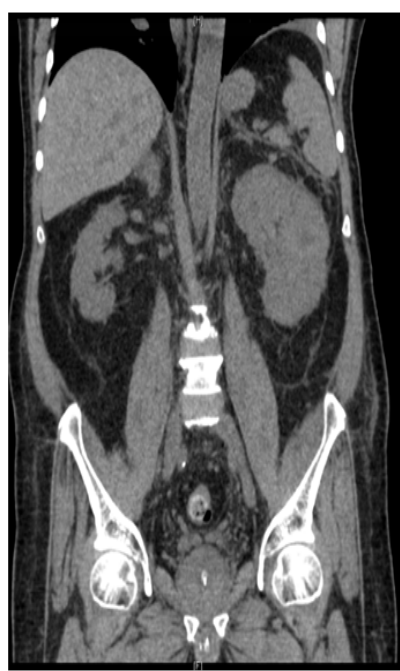

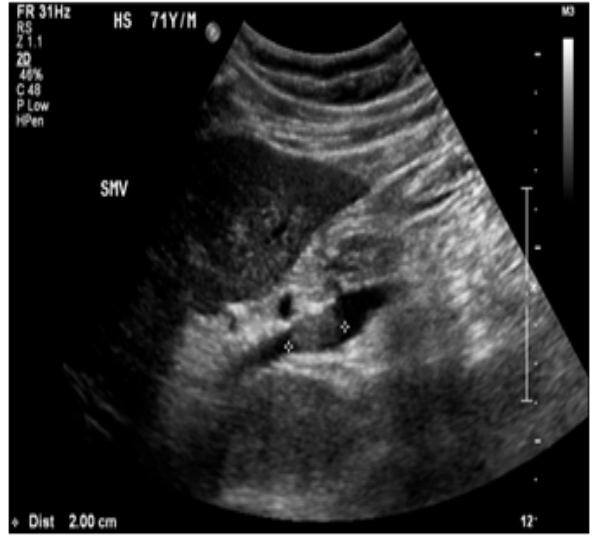

Figure 3. Non-enhanced CT showing and enlargement of the left renal vein and IVC.

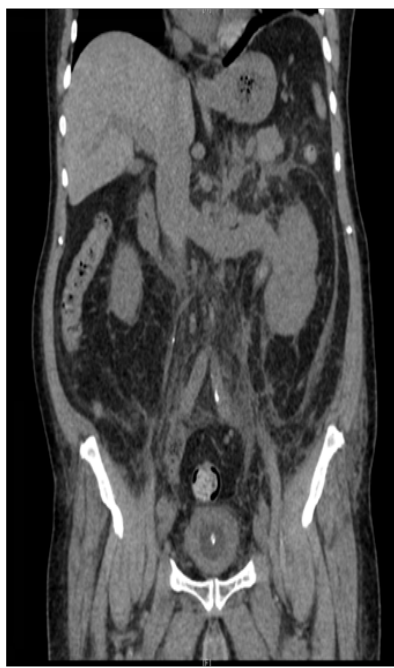


use. Other reasons for this rise in incidence could be the increased diagnosis of diabetes mellitus, hypertension, and obesity in the Saudi population [4-6], which are all proven risk factors for renal cancer [7].

It is estimated that around 62,700 new cases of kidney cancer will be diagnosed in the United States in 2016; the majority being discovered incidentally through abdominal imaging done for various other unrelated reasons [8]. The gold standard for the diagnosis of renal malignancy is enhanced computed tomography.

In our case the finding of a renal vein and IVC thrombus, which after biopsy proved to be of renal origin, pointed us towards the diagnosis of RCC. This is due to the fact that IVC thrombus is considered one of the hallmarks for RCC, presenting in 10-25\% of cases [9]. Coupled with the fact that an IVC thrombus in an upper tract TCC has rarely ever been reported, we were confident in proceeding with our diagnosis of RCC. It was much to our surprise when the pathology report showed a transitional cell carcinoma of the upper tract rather than a RCC. Being one of the few ever reported cases of upper tract TCC with IVC thrombus in the world, and the first reported one in the kingdom of Saudi Arabia; we hope that this report can add some light on the nature of upper tract TCC and the vascular invasion which it can present with.

\section{References}

[1]. Lipworth L, Tarone RE, McLaughlin JK. The epidemiology of renal cell carcinoma. J Urol. 2006 Dec;176(6 Pt 1): 235.

[2]. Haferkamp A, Bastian PJ, Jakobi H, Pritsch M, Pfitzenmaier J, Albers P, et al. Renal cell carcinoma with tumor thrombus extension into the vena cava: prospective long-term followup. J Urol. 2007 May;177(5):1703-1708. 10.1016/j.juro.2007.01.039.

[3]. Alkhateeb SS, Alkhateeb JM, Alrashidi EA. Increasing trends in kidney cancer over the last 2 decades in Saudi Arabia. Saudi Med J. 2015 Jun;36(6):698703. doi:10.15537/smj.2015.6.10841.

[4]. Alqurashi KA, Aljabri KS, Bokhari SA. Prevalence of diabetes mellitus in a Saudi community. Ann Saudi Med. 2011 Feb;31(1):19-23. doi:10.4103/0256-4947.75773.

[5]. Al-Nozha MM, Abdullah M, Arafah MR, Khalil MZ, Khan NB, Al-Mazrou YY, et al. Hypertension in Saudi Arabia. Saudi Med J. 2007;28(1):77-84.

[6]. Al-Nozha MM, Al-Mazrou YY, Al-Maatouq MA, Arafah MR, Khalil MZ, Khan NB, et al. Obesity in Saudi Arabia. Saudi Med J. 2005 May;26(5):8249.

[7]. Washio M, Mori M, Mikami K, Miki T, Watanabe Y, Nakao M, et al. Risk factors for renal cell carcinoma in a Japanese population. Asian Pac J Cancer Prev. 2014;15(21):9065-9070.

[8]. American Cancer Society. Cancer Facts and Figures 2016. Atlanta, GA: American Cancer Society; 2016.

[9]. Psutka, Sarah P, Bradley C Leibovich. Management of Inferior Vena Cava Tumor Thrombus in Locally Advanced Renal Cell Carcinoma. Ther Adv Urol. 2015 Aug;7(4):216-229.

[10]. Agha RA, Fowler AJ, Saetta A, Barai I, Rajmohan S, Orgill DP, et al. The SCARE Statement: Consensus-based surgical case report guidelines. Int J Surg. 2016 Oct;34:180-186. 